\title{
Bäcklund Transformation of Fractional Riccati Equation and Infinite Sequence Solutions of Nonlinear Fractional PDEs
}

\author{
Bin Lu \\ School of Mathematical Sciences, Anhui University, Hefei 230601, China \\ Correspondence should be addressed to Bin Lu; lubinhb@163.com
}

Received 24 October 2013; Accepted 9 December 2013; Published 5 January 2014

Academic Editor: Fuding Xie

Copyright (C) 2014 Bin Lu. This is an open access article distributed under the Creative Commons Attribution License, which permits unrestricted use, distribution, and reproduction in any medium, provided the original work is properly cited.

The Bäcklund transformation of fractional Riccati equation with nonlinear superposition principle of solutions is employed to establish the infinite sequence solutions of nonlinear fractional partial differential equations in the sense of modified RiemannLiouville derivative. To illustrate the reliability of the method, some examples are provided.

\section{Introduction}

Recently, nonlinear fractional differential equations increasingly are used to describe nonlinear phenomena in fluid mechanics, biology, engineering, physics, and other areas of science [1-3]. Much efforts have been spent in recent years to develop various techniques to deal with fractional differential equations. However, for the nonlinear differential equations including fractional calculus, the analytical or numerical results are usually difficult to be obtained. It is therefore needed to find a proper method to solve the problem of nonlinear differential equations containing fractional calculus.

In the past, several methods have been formulated, such as Adomian decomposition method $[4,5]$, variational iteration method $[6,7]$, homotopy perturbation method $[8$, 9], differential transform method [10, 11], and fractional subequation method [12-14]. S. Zhang and H.-Q. Zhang [12] first proposed a new direct method called fractional subequation method in solving nonlinear time fractional biological population model and $(4+1)$-dimensional space-time fractional Fokas equation, based on the homogeneous balance principle and Jumarie's modified Riemann-Liouville derivative.

In this paper, based on the Bäcklund transformation technique and the known seed solutions, we will devise effective way for solving fractional partial differential equations. It will be shown that the use of the Bäcklund transformation allows us to obtain new exact solutions from the known seed solutions.

\section{Bäcklund Transformation of the Fractional Riccati Equation and Nonlinear Superposition Principle}

Firstly, we give some definitions and properties of the modified Riemann-Liouville derivative [15] which are used in this paper.

Assume that $f: R \rightarrow R, x \rightarrow f(x)$ denote a continuous (but not necessarily differentiable) function, and let $h$ denote a constant discretization span. Jumarie defined the fractional derivative in the limit form

$$
f^{\alpha}(x):=\lim _{h \downarrow 0} \frac{\Delta^{\alpha}[f(x)-f(0)]}{h^{\alpha}}, \quad 0<\alpha<1,
$$

where

$$
\begin{aligned}
\Delta^{\alpha} f(x)= & \sum_{k=0}^{\infty}(-1)^{k} \frac{\Gamma(1+\alpha)}{\Gamma(1+k) \Gamma(\alpha-k+1)} \\
& \times f[x+(\alpha-k) h] .
\end{aligned}
$$

This definition is close to the standard definition of the derivative (calculus for beginners), and as a direct result, the 
$\alpha$ th derivative of a constant, $0<\alpha<1$, is zero. An alternative, which is strictly equivalent to (1) is as follows:

$$
\begin{array}{r}
f^{\alpha}(x):=\frac{1}{\Gamma(1-\alpha)} \frac{d}{d x} \int_{0}^{x}(x-\xi)^{-\alpha}[f(\xi)-f(0)] d \xi \\
0<\alpha<1, \\
f^{\alpha}(x):=\left(f^{(n)}(x)\right)^{(\alpha-n)}, \quad n \leq \alpha \leq n+1, n \geq 1 .
\end{array}
$$

Some properties of the fractional modified RiemannLiouville derivative that were summarized in four useful formulas of them are

$$
\begin{gathered}
D_{x}^{\alpha} x^{\gamma}=\frac{\Gamma(1+\gamma)}{\Gamma(1+\gamma-\alpha)} x^{\gamma-\alpha}, \quad \gamma>0, \\
D_{x}^{\alpha}(u(x) v(x))=v(x) D_{x}^{\alpha} u(x)+u(x) D_{x}^{\alpha} v(x), \\
D_{x}^{\alpha}[f(u(x))]=f_{u}^{\prime}(u) D_{x}^{\alpha} u(x), \\
D_{x}^{\alpha}[f(u(x))]=D_{u}^{\alpha} f(u)\left(u_{x}^{\prime}\right)^{\alpha},
\end{gathered}
$$

which are direct consequences of the equality $d^{\alpha} x(t)=\Gamma(1+$ $\alpha) d x(t)$ which holds for nondifferentiable functions. In the above formulas (5)-(6), $u(x)$ is nondifferentiable function in (5) and (6) and differentiable in (7), $v(x)$ is nondifferentiable, and $f(u)$ is differentiable in (6) and nondifferentiable in (7).

Recall the fractional Riccati equation:

$$
D_{\xi}^{\alpha} \phi(\xi)=\sigma+\phi^{2}(\xi), \quad 0<\alpha \leq 1,
$$

S. Zhang and H.-Q. Zhang [12] derived some exact solutions to (8) as follows:

$$
\phi(\xi)= \begin{cases}-\sqrt{-\sigma} \tanh _{\alpha}(\sqrt{-\sigma} \xi), & \sigma<0, \\ -\sqrt{-\sigma} \operatorname{coth}_{\alpha}(\sqrt{-\sigma} \xi), & \sigma<0, \\ \sqrt{\sigma} \tan _{\alpha}(\sqrt{\sigma} \xi), & \sigma>0, \\ -\sqrt{\sigma} \cot _{\alpha}(\sqrt{\sigma} \xi), & \sigma>0, \\ -\frac{\Gamma(1+\alpha)}{\xi^{\alpha}+\omega}, & \omega=\text { const }, \sigma=0,\end{cases}
$$

where the generalized hyperbolic and trigonometric functions are defined as

$$
\begin{gathered}
\sin _{\alpha}(\xi)=\frac{E_{\alpha}\left(i \xi^{\alpha}\right)-E_{\alpha}\left(-i \xi^{\alpha}\right)}{2 i}, \\
\cos _{\alpha}(\xi)=\frac{E_{\alpha}\left(i \xi^{\alpha}\right)+E_{\alpha}\left(-i \xi^{\alpha}\right)}{2 i}, \\
\tan _{\alpha}(\xi)=\frac{\sin _{\alpha}(\xi)}{\cos _{\alpha}(\xi)}, \quad \cot _{\alpha}(\xi)=\frac{\cos _{\alpha}(\xi)}{\sin _{\alpha}(\xi)}, \\
\sinh _{\alpha}(\xi)=\frac{E_{\alpha}\left(\xi^{\alpha}\right)-E_{\alpha}\left(-\xi^{\alpha}\right)}{2} \\
\cosh _{\alpha}(\xi)=\frac{E_{\alpha}\left(\xi^{\alpha}\right)+E_{\alpha}\left(-\xi^{\alpha}\right)}{2} \\
\tanh _{\alpha}(\xi)=\frac{\sinh _{\alpha}(\xi)}{\cosh _{\alpha}(\xi)}, \quad \operatorname{coth}_{\alpha}(\xi)=\frac{\cosh _{\alpha}(\xi)}{\sinh _{\alpha}(\xi)}
\end{gathered}
$$

where $E_{\alpha}(\xi)=\sum_{k=0}^{\infty}\left(\xi^{k} / \Gamma(1+k \alpha)\right)(\alpha>0)$ is the MittagLeffler function.

Next, we introduce the Bäcklund transformation of fractional Riccati equation (8):

$$
\bar{\phi}(\xi)=\frac{A_{1}+A_{2} \phi(\xi)+A_{3} \phi(\xi)^{2}+A_{4} \phi(\xi)^{3}+A_{5} \phi(\xi)^{4}}{B_{1}+B_{2} \phi(\xi)+B_{3} \phi(\xi)^{2}+B_{4} \phi(\xi)^{3}+B_{5} \phi(\xi)^{4}}
$$

that is, $\bar{\phi}(\xi)$ satisfies the fractional Riccati equation

$$
D_{\xi}^{\alpha} \bar{\phi}(\xi)=\sigma+\bar{\phi}^{2}(\xi)
$$

where $A_{i}(i=1, \ldots, 4), B_{1}$ are arbitrary parameters, $A_{5}=$ $\left(A_{2} B_{1}^{2}-B_{1}^{3}-A_{1} A_{3} B_{1}+A_{1}^{2} A_{4}\right) B_{1} / A_{1}^{3}, B_{2}=\left(\sigma\left(A_{2} B_{1}-B_{1}^{2}\right)-\right.$ $\left.A_{1}^{2}\right) / \sigma A_{1}, B_{3}=\left(\sigma\left(B_{1}^{3}-A_{2} B_{1}^{2}+A_{1} A_{3} B_{1}\right)+B_{1} A_{1}^{2}-A_{1}^{2} A_{2}\right) / \sigma A_{1}^{2}$, $B_{4}=\left(\sigma\left(B_{1}^{3} A_{2}-B_{1}^{4}-A_{1} A_{3} B_{1}^{2}+A_{1}^{2} A_{4} B_{1}\right)+B_{1} A_{1}^{2} A_{2}-A_{1}^{2} B_{1}^{2}-\right.$ $\left.A_{1}^{3} A_{3}\right) / \sigma A_{1}^{3}, B_{5}=\left(A_{1} A_{3} B_{1}-A_{1}^{2} A_{4}+B_{1}^{3}-A_{2} B_{1}^{2}\right) / \sigma A_{2}^{2}$ and $\phi(\xi)$ are the known solutions (9).

Specially, if we take $A_{3}=A_{4}=A_{5}=B_{3}=B_{4}=B_{5}=$ 0 in (11), the Bäcklund transformation of factional Riccati equation can be obtained as

$$
\bar{\phi}(\xi)=\frac{-\sigma A_{1}+B_{1} \phi(\xi)}{B_{1}+A_{1} \phi(\xi)} .
$$

By means of solutions $\phi(\xi)(9)$, we can construct the following infinite sequence exact solutions of fractional Riccati equation (8). Here in the following cases we given several Bäcklund transformations of solutions.

Case 1. When $\sigma<0$, if $\phi_{k-1}(\xi)$ is the solution of fractional Riccati equation (8), then the following $\phi_{k}(\xi)$ are also the solutions of (8):

$$
\begin{aligned}
& \phi_{k}(\xi) \\
& =\frac{A_{1}+A_{2} \phi_{k-1}(\xi)+A_{3} \phi_{k-1}(\xi)^{2}+A_{4} \phi_{k-1}(\xi)^{3}+A_{5} \phi_{k-1}(\xi)^{4}}{B_{1}+B_{2} \phi_{k-1}(\xi)+B_{3} \phi_{k-1}(\xi)^{2}+B_{4} \phi_{k-1}(\xi)^{3}+B_{5} \phi_{k-1}(\xi)^{4}} \\
& \phi_{0}(\xi)=-\sqrt{-\sigma} \tanh _{\alpha}[\sqrt{-\sigma}(\xi)]
\end{aligned}
$$

Case 2. When $\sigma<0$, if $\phi_{k-1}(\xi)$ is the solution of fractional Riccati equation (8), then the following $\phi_{k}(\xi)$ are also the solutions of (8):

$$
\begin{aligned}
& \phi_{k}(\xi) \\
& =\frac{A_{1}+A_{2} \phi_{k-1}(\xi)+A_{3} \phi_{k-1}(\xi)^{2}+A_{4} \phi_{k-1}(\xi)^{3}+A_{5} \phi_{k-1}(\xi)^{4}}{B_{1}+B_{2} \phi_{k-1}(\xi)+B_{3} \phi_{k-1}(\xi)^{2}+B_{4} \phi_{k-1}(\xi)^{3}+B_{5} \phi_{k-1}(\xi)^{4}} \\
& \phi_{0}(\xi)=-\sqrt{-\sigma} \operatorname{coth}_{\alpha}[\sqrt{-\sigma}(\xi)]
\end{aligned}
$$


Case 3. When $\sigma>0$, if $\phi_{k-1}(\xi)$ is the solution of fractional Riccati equation (8), then the following $\phi_{k}(\xi)$ are also the solutions of (8):

$$
\begin{gathered}
\phi_{k}(\xi) \\
=\frac{A_{1}+A_{2} \phi_{k-1}(\xi)+A_{3} \phi_{k-1}(\xi)^{2}+A_{4} \phi_{k-1}(\xi)^{3}+A_{5} \phi_{k-1}(\xi)^{4}}{B_{1}+B_{2} \phi_{k-1}(\xi)+B_{3} \phi_{k-1}(\xi)^{2}+B_{4} \phi_{k-1}(\xi)^{3}+B_{5} \phi_{k-1}(\xi)^{4}} \\
\phi_{0}(\xi)=\sqrt{\sigma} \tan _{\alpha}[\sqrt{\sigma}(\xi)] .
\end{gathered}
$$

Case 4. When $\sigma>0$, if $\phi_{k-1}(\xi)$ is the solution of fractional Riccati equation (8), then the following $\phi_{k}(\xi)$ are also the solutions of (8):

$$
\begin{gathered}
\phi_{k}(\xi) \\
=\frac{A_{1}+A_{2} \phi_{k-1}(\xi)+A_{3} \phi_{k-1}(\xi)^{2}+A_{4} \phi_{k-1}(\xi)^{3}+A_{5} \phi_{k-1}(\xi)^{4}}{B_{1}+B_{2} \phi_{k-1}(\xi)+B_{3} \phi_{k-1}(\xi)^{2}+B_{4} \phi_{k-1}(\xi)^{3}+B_{5} \phi_{k-1}(\xi)^{4}} \\
\phi_{0}(\xi)=-\sqrt{\sigma} \cot _{\alpha}[\sqrt{\sigma}(\xi)]
\end{gathered}
$$

where $A_{i}(i=1, \ldots, 4), B_{1}$ are arbitrary parameters, $A_{5}=$ $\left(A_{2} B_{1}^{2}-B_{1}^{3}-A_{1} A_{3} B_{1}+A_{1}^{2} A_{4}\right) B_{1} / A_{1}^{3}, B_{2}=\left(\sigma\left(A_{2} B_{1}-B_{1}^{2}\right)-\right.$ $\left.A_{1}^{2}\right) / \sigma A_{1}, B_{3}=\left(\sigma\left(B_{1}^{3}-A_{2} B_{1}^{2}+A_{1} A_{3} B_{1}\right)+B_{1} A_{1}^{2}-A_{1}^{2} A_{2}\right) / \sigma A_{1}^{2}$, $B_{4}=\left(\sigma\left(B_{1}^{3} A_{2}-B_{1}^{4}-A_{1} A_{3} B_{1}^{2}+A_{1}^{2} A_{4} B_{1}\right)+B_{1} A_{1}^{2} A_{2}-A_{1}^{2} B_{1}^{2}-\right.$ $\left.A_{1}^{3} A_{3}\right) / \sigma A_{1}^{3}$, and $B_{5}=\left(A_{1} A_{3} B_{1}-A_{1}^{2} A_{4}+B_{1}^{3}-A_{2} B_{1}^{2}\right) / \sigma A_{2}^{2}$.

Case 5. When $\sigma=0$, if $\phi_{k-1}(\xi)$ is the solution of fractional Riccati equation (8), then the following $\phi_{k}(\xi)$ are also the solutions of (8):

$$
\begin{gathered}
\phi_{k}(\xi)=\frac{B_{1} \phi_{k-1}(\xi)}{B_{1}+B_{2} \phi_{k-1}(\xi)}, \\
\phi_{0}(\xi)=-\frac{\Gamma(1+\alpha)}{\xi^{\alpha}+\omega},
\end{gathered}
$$

where $B_{1}, B_{2}$ are arbitrary constant, and $B_{2} \neq 0$.

Nonlinear Superposition Principle. (1) If $\phi_{k-1}(\xi), \phi_{k-2}(\xi)$ are the solutions of fractional Riccati equation (8), respectively, then the following $\phi_{k}(\xi)(k=2,3, \ldots)$ are also the solutions of (8) which read

$$
\begin{aligned}
\phi_{k}(\xi)=\left(\sigma \sqrt{-\sigma}\left(-2 a_{1}+a_{2}\right)-a_{1}\left(\phi_{k-1}(\xi)+\phi_{k-2}(\xi)\right)\right. \\
\left.+a_{2} \sqrt{-\sigma} \phi_{k-1}(\xi) \phi_{k-2}(\xi)\right) \\
\times\left(-a_{2}+a_{1} \sqrt{-\sigma}\left(\phi_{k-1}(\xi) \phi_{k-2}(\xi)\right)\right. \\
\left.+\left(-2 a_{1}+a_{2}\right) \phi_{k-1}(\xi) \phi_{k-2}(\xi)\right)^{-1},
\end{aligned}
$$

where $a_{1}, a_{2}$ are arbitrary nonzero constants.

(2) If $\phi_{k-1}(\xi), \phi_{k-2}(\xi)$, and $\phi_{k-3}(\xi)$ are the solutions of fractional Riccati equation (8), respectively, then the following $\phi_{k}(\xi)(k=2,3, \ldots)$ are also the solutions of $(8)$ which read

$$
\begin{aligned}
\phi_{k}(\xi)= & \left(\phi_{k-1}(\xi)\left(\phi_{k-3}(\xi)-\phi_{k-2}(\xi)\right)\right. \\
& \left.-c \phi_{k-2}(\xi)\left(\phi_{k-3}(\xi)-\phi_{k-1}(\xi)\right)\right) \\
& \times\left(\phi_{k-3}(\xi)-\phi_{k-2}(\xi)\right. \\
& \left.-c\left(\phi_{k-3}(\xi)-\phi_{k-1}(\xi)\right)\right)^{-1},
\end{aligned}
$$

where $c$ is an arbitrary nonzero constant.

Applying the nonlinear superposition formulas (19)-(20), we can obtain the following new infinite sequence exact solutions of fractional Riccati equation (8). For example, when $\sigma<0$, we can get the infinite sequence new solutions $\widetilde{\phi}_{l}(\xi)(l=2,3, \ldots)$ as follows:

$$
\begin{gathered}
\tilde{\phi}_{l}(\xi)=\phi_{k}(\xi) \\
=\left(\sigma \sqrt{-\sigma}\left(-2 a_{1}+a_{2}\right)-a_{1}\left(\phi_{k-1}(\xi)+\phi_{k-2}(\xi)\right)\right. \\
\left.+a_{2} \sqrt{-\sigma} \phi_{k-1}(\xi) \phi_{k-2}(\xi)\right) \\
\times\left(-a_{2}+a_{1} \sqrt{-\sigma}\left(\phi_{k-1}(\xi) \phi_{k-2}(\xi)\right)\right. \\
\left.+\left(-2 a_{1}+a_{2}\right) \phi_{k-1}(\xi) \phi_{k-2}(\xi)\right)^{-1}, \\
\phi_{0}(\xi)=-\sqrt{-\sigma} \tanh _{\alpha}[\sqrt{-\sigma}(\xi)] \\
\phi_{k}(\xi)=\left(A_{1}+A_{2} \phi_{k-1}(\xi)+A_{3} \phi_{k-1}(\xi)^{2}\right. \\
\left.\quad+A_{4} \phi_{k-1}(\xi)^{3}+A_{5} \phi_{k-1}(\xi)^{4}\right) \\
\times\left(B_{1}+B_{2} \phi_{k-1}(\xi)+B_{3} \phi_{k-1}(\xi)^{2}\right. \\
\left.+B_{4} \phi_{k-1}(\xi)^{3}+B_{5} \phi_{k-1}(\xi)^{4}\right)^{-1}
\end{gathered}
$$

where $a_{1}, a_{2}$ are arbitrary nonzero constants.

\section{Summary of the Method}

In this section, we describe the main steps of the fractional subequation method for finding exact solutions of fractional differential equations.

Let us consider the fractional differential equation with independent variables $x=\left(x_{1}, x_{2}, \ldots, x_{m}, t\right)$ and dependent variable $u$,

$$
F\left(u, u_{t}, u_{x_{1}}, u_{x_{2}}, u_{x_{3}}, D_{t}^{\alpha} u, D_{x_{1}}^{\alpha} u, D_{x_{2}}^{\alpha} u, D_{x_{3}}^{\alpha} u, \ldots\right)=0
$$

where $D_{t}^{\alpha} u, D_{x_{1}}^{\alpha} u, D_{x_{2}}^{\alpha} u$, and $D_{x_{3}}^{\alpha} u$ are the modified RiemannLiouville derivatives of $u$ with respect to $t, x_{1}, x_{2}$, and $x_{3}$, respectively.

Step 1. Using the variable transformation

$$
\begin{gathered}
u\left(x_{1}, x_{2}, \ldots, x_{m}, t\right)=u(\xi), \\
\xi=x_{1}+l_{1} x_{2}+\cdots+l_{m-1} x_{m}+\lambda t+\xi_{0}
\end{gathered}
$$


where $l_{i}(i=1, \ldots, m-1)$ and $\lambda$ are constants to be determined later, the fractional differential equation (24) is reduced to a nonlinear fractional ordinary differential equation

$$
H\left(u(\xi), u^{\prime}(\xi), u^{\prime \prime}(\xi), D_{\xi}^{\alpha} u(\xi), \ldots\right)=0,
$$

where $" \prime \prime "=d / d(\xi)$.

Step 2. We suppose that (26) has the following solution:

$$
u(\xi)=\sum_{j=0}^{n} a_{j} \psi^{j}(\xi)
$$

where $a_{j}(j=0, \ldots, n)$ are constants to be determined, positive integer $n$ can be determined by balancing the highest order derivatives and nonlinear terms in (24) or (26), and $\psi(\xi)$ comes from the following Bäcklund transformation for the fractional Riccati equation:

$$
\psi(\xi)=\frac{A_{1}+A_{2} \phi(\xi)+A_{3} \phi(\xi)^{2}+A_{4} \phi(\xi)^{3}+A_{5} \phi(\xi)^{4}}{B_{1}+B_{2} \phi(\xi)+B_{3} \phi(\xi)^{2}+B_{4} \phi(\xi)^{3}+B_{5} \phi(\xi)^{4}}
$$

that is, $\psi(\xi)$ satisfies the fractional Riccati equation

$$
D_{\xi}^{\alpha} \psi(\xi)=\sigma+\psi^{2}(\xi), \quad 0<\alpha \leq 1,
$$

where $\phi(\xi)$ are the known solutions of (8).

Step 3. Substituting the explicit formal solution (27) into (26) and setting the coefficients of the powers of $\psi_{i}(\xi)$ to be zero, we obtain an overdetermined nonlinear algebraic system in $a_{j}(j=0, \ldots, n), l_{i}(i=1, \ldots, m-1)$, and $\lambda$.

Step 4. Solving the nonlinear algebraic system yields the explicit expressions of the parameters $a_{j}(j=0, \ldots, n), l_{i}(i=$ $1, \ldots, m-1)$, and $\lambda$. Then substituting these values into (27), we may obtain the exact solutions of the nonlinear fractional differential equation (24).

\section{Applications of the Method}

In this section, we present two examples to illustrate the applicability of the our method to solve nonlinear fractional partial differential equations.

Example 1. We first consider the space-time fractional bidirectional wave equations in the form [16]

$$
\begin{aligned}
& D_{t}^{\alpha} v+D_{x}^{\alpha} u+u D_{x}^{\alpha} v+v D_{x}^{\alpha} u \\
& \quad+a D_{x}^{\alpha} D_{x}^{\alpha} D_{x}^{\alpha} u-b D_{x}^{\alpha} D_{x}^{\alpha} D_{t}^{\alpha} v=0, \quad 0<\alpha \leq 1, \\
& D_{t}^{\alpha} u+D_{x}^{\alpha} v+u D_{x}^{\alpha} u \\
& \quad+c D_{x}^{\alpha} D_{x}^{\alpha} D_{x}^{\alpha} v-d D_{x}^{\alpha} D_{x}^{\alpha} D_{t}^{\alpha} u=0,
\end{aligned}
$$

where $x$ represents the distance along the channel, $t$ is the elapsed time, the variable $v(x, t)$ is the dimensionless deviation of the water surface from its undisturbed position, $u(x, t)$ is the dimensionless horizontal velocity, $a, b, c$ are real constants. When $\alpha=1,(30)$ is the generalization of bidirectional wave equations, which can be used as a model equation for the propagation of long waves on the surface of water with a small amplitude.

For our purpose, we introduce the following transformations:

$$
u(x, t)=u(\xi), \quad v(x, t)=v(\xi), \quad \xi=x+\lambda t+\xi_{0},
$$

where $\lambda$ is constant.

Substituting (32) into (30), we can know that (30) is reduced into a fractional ordinary differential equations:

$$
\begin{aligned}
\lambda^{\alpha} D_{\xi}^{\alpha} v & +D_{\xi}^{\alpha} u+u D_{\xi}^{\alpha} v+v D_{\xi}^{\alpha} u \\
& +a D_{\xi}^{\alpha} D_{\xi}^{\alpha} D_{\xi}^{\alpha} u-b \lambda^{\alpha} D_{\xi}^{\alpha} D_{\xi}^{\alpha} D_{\xi}^{\alpha} v=0, \quad 0<\alpha \leq 1, \\
\lambda^{\alpha} D_{\xi}^{\alpha} u & +D_{\xi}^{\alpha} v+u D_{\xi}^{\alpha} u \\
& +c D_{\xi}^{\alpha} D_{\xi}^{\alpha} D_{\xi}^{\alpha} v-d \lambda^{\alpha} D_{\xi}^{\alpha} D_{\xi}^{\alpha} D_{\xi}^{\alpha} u=0 .
\end{aligned}
$$

We suppose that (33) has the solution in the form

$$
\begin{aligned}
& u(\xi)=a_{0}+\sum_{i=1}^{n} a_{i} \phi(\xi)^{i}, \\
& v(\xi)=b_{0}+\sum_{j=1}^{m} b_{j} \phi(\xi)^{j} .
\end{aligned}
$$

Balancing the highest order derivative terms and nonlinear terms in (33), we have $m=n=2$. Substituting (35) given the value of $n=2$ and $m=2$ along with (8) into (33) and then setting the coefficients of $\phi(\xi)$ to zero, we can obtain a set of algebraic equations about $a_{0}, a_{1}, a_{2}, b_{0}, b_{1}, b_{2}$, and $\lambda$. Solving the algebraic equations by Maple, we have,

$$
\begin{gathered}
a_{0}=-\lambda^{\alpha}-\frac{12 d \lambda^{\alpha}-a_{2}(1+8 c \sigma)}{12 c}, \quad a_{1}=0, \\
b_{0}=-1-4 a \sigma-\frac{\left(a_{2}-12 \lambda^{\alpha} d\right)(2 d+8 b c \sigma-b) \lambda^{\alpha}}{24 c^{2}}, \\
b_{1}=0, \quad b_{2}=-6 a+\frac{b \lambda^{\alpha}\left(12 d \lambda^{\alpha}-a_{2}\right)}{2 c},
\end{gathered}
$$

where $\lambda, a_{2}$ are arbitrary constants.

Substituting the above result into (35), we obtain new types of exact solutions of (30) as follows:

$$
\begin{gathered}
u_{k}(\xi)=-\lambda^{\alpha}-\frac{12 d \lambda^{\alpha}-a_{2}(1+8 c \sigma)}{12 c}+a_{2} \phi_{k}(\xi)^{2}, \\
v_{k}(\xi)=-1-4 a \sigma-\frac{\left(a_{2}-12 \lambda^{\alpha} d\right)(2 d+8 b c \sigma-b) \lambda^{\alpha}}{24 c^{2}} \\
-6 a+\frac{b \lambda^{\alpha}\left(12 d \lambda^{\alpha}-a_{2}\right)}{2 c} \phi_{k}(\xi)^{2}, \quad k=0,1, \ldots
\end{gathered}
$$

The expression $\phi_{k}(\xi)$ appearing in these solutions is given by relations (14)-(18) and the nonlinear superposition formulas (19)-(20), where $\xi=x+\lambda t+\xi_{0}, a_{2}, \lambda$, and $\sigma$ are real constants. 
Example 2. We consider the following space-time fractional Sharma-Tasso-Olver (STO) equation [17] in the form

$$
\begin{aligned}
D_{t}^{\alpha} u & +3 a\left(D_{x}^{\alpha} u\right)^{2}+3 a u^{2} D_{x}^{\alpha} u+3 a u D_{x}^{\alpha} D_{x}^{\alpha} u \\
& +a D_{x}^{\alpha} D_{x}^{\alpha} D_{x}^{\alpha} u=0, \quad 0<\alpha \leq 1,
\end{aligned}
$$

where $a$ is an arbitrary constant and $\alpha$ is a parameter describing the order of the fractional derivative. When $\alpha=1$, (38) is the generalization of classical nonlinear STO equation, which was first derived as an example of odd members of Burgers hierarchy by Tasso.

For our purpose, we introduce the following transformations:

$$
u(x, t)=u(\xi), \quad \xi=x+\lambda t+\xi_{0}
$$

where $\lambda$ is constant.

Substituting (39) into (38), we can know that (38) is reduced into a fractional ordinary differential equation:

$$
\begin{aligned}
\lambda^{\alpha} D_{\xi}^{\alpha} u & +3 a\left(D_{\xi}^{\alpha} u\right)^{2}+3 a u^{2} D_{\xi}^{\alpha} u \\
& +3 a u D_{\xi}^{\alpha} D_{\xi}^{\alpha} u+a D_{\xi}^{\alpha} D_{\xi}^{\alpha} D_{\xi}^{\alpha} u=0 .
\end{aligned}
$$

We suppose that (40) has the solution in the form

$$
u(\xi)=a_{0}+\sum_{i=1}^{n} a_{i} \phi(\xi)^{i}
$$

Balancing $u D_{\xi}^{\alpha} D_{\xi}^{\alpha} u$ and $D_{\xi}^{\alpha} D_{\xi}^{\alpha} D_{\xi}^{\alpha} u$, we have $2 n+2=n+$ $3 \Rightarrow n=1$. Substituting (41) given the value of $n=1$ along with (8) into (40) and then setting the coefficients of $\phi(\xi)$ to zero, we can obtain a set of algebraic equations about $a_{0}, a_{1}$, and $\lambda$. Solving the algebraic equations by Maple, we have

$$
a_{0}=0, \quad a_{1}=-2, \quad \sigma=\frac{\lambda^{\alpha}}{4 a},
$$

where $\lambda$ is an arbitrary constant, and

$$
a_{1}=-1, \quad \sigma=\frac{\lambda^{\alpha}+3 a a_{0}^{2}}{a},
$$

where $a_{0}, \lambda$ are arbitrary constants.

Substituting the above results into (41), we obtain new types of exact solutions of (38) as follows:

$$
u_{1, k}(x, t)=-2 \phi_{k}(\xi), \quad k=0,1, \ldots
$$

The expression $\phi_{k}(\xi)$ appearing in these solutions is given by relations (14)-(18) and the nonlinear superposition formulas (19)-(20), where $\sigma=\lambda^{\alpha} / 4 a, \xi=x+\lambda t+\xi_{0}$, and

$$
u_{2, k}(x, t)=a_{0}-\phi_{k}(\xi), \quad k=0,1, \ldots
$$

The expression $\phi_{k}(\xi)$ appearing in these solutions is given by relations (14)-(18) and the nonlinear superposition formulas (19)-(20), where $\sigma=\left(\lambda^{\alpha}+3 a a_{0}^{2}\right) / a$ and $\xi=x+\lambda t+\xi_{0}$.

\section{Conclusion}

Bäcklund transformation of the fractional Riccati equation with nonlinear superposition principle of known solutions is applied successfully for solving the system of nonlinear fractional differential equation. To the best of our knowledge, the solutions obtained in this paper have not been reported in the literature. It can be concluded that this method is very simple and reliable and proposes a variety of exact solutions to fractional differential equations.

\section{Conflict of Interests}

The author declares that there is no conflict of interests regarding the publication of this paper.

\section{Acknowledgments}

The author would like to express sincere thanks to referees for their valuable suggestions and comments. The project are supported by the 211 Project and Start-up Research Funds for Doctors of Anhhui University (02303319-33190074), the Key Foundation of Anhui Education Bureau (KJ2013A028, KJ2012A019), the Research Fund for the Doctoral Program of Higher Education (20103401120002), and 211 Project of Anhui University (02303303-33030011).

\section{References}

[1] K. S. Miller and B. Ross, An Introduction To the Fractional Calculus and Fractional Differential Equations, Wiley, New York, NY, USA, 1993.

[2] A. A. Kilbas, H. M. Srivastava, and J. J. Trujillo, Theory and Applications of Fractional Differential Equations, Elsevier, San Diego, Calif, USA, 2006.

[3] I. Podlubny, Fractional Differential Equations, Academic Press, San Diego, Calif, USA, 1999.

[4] J. S. Duan, C. Temuer, and R. Randolph, “The Adomian decomposition method with convergence acceleration techniques for nonlinear fractional differential equations," Computers \& Mathematics with Applications, vol. 66, no. 5, pp. 728-736, 2013.

[5] A. M. A. El-Sayed and M. Gaber, "The Adomian decomposition method for solving partial differential equations of fractal order in finite domains," Physics Letters A, vol. 359, no. 3, pp. 175-182, 2006.

[6] Z. Odibat and S. Momani, "The variational iteration method: an efficient scheme for handling fractional partial differential equations in fluid mechanics," Computers and Mathematics with Applications, vol. 58, no. 11-12, pp. 2199-2208, 2009.

[7] G.-C. Wu and E. W. M. Lee, "Fractional variational iteration method and its application," Physics Letters A, vol. 374, no. 25, pp. 2506-2509, 2010.

[8] A. A. Elbeleze, A. Kilicman, and B. M. Taib, "Homotopy perturbation method for fractional black-scholes European option pricing equations using sumudu transform," Mathematical Problems in Engineering, vol. 2013, Article ID 524852, 7 pages, 2013.

[9] A. M. A. El-Sayed, A. Elsaid, I. L. El-Kalla, and D. Hammad, "A homotopy perturbation technique for solving partial differential equations of fractional order in finite domains," Applied 
Mathematics and Computation, vol. 218, no. 17, pp. 8329-8340, 2012.

[10] S. Momani, Z. Odibat, and V.S. Erturk, "Generalized differential transform method for solving a space- and time-fractional diffusion-wave equation," Physics Letters A, vol. 370, no. 5-6, pp. 379-387, 2007.

[11] Z. Odibat and S. Momani, "A generalized differential transform method for linear partial differential equations of fractional order," Applied Mathematics Letters, vol. 21, no. 2, pp. 194-199, 2008.

[12] S. Zhang and H.-Q. Zhang, "Fractional sub-equation method and its applications to nonlinear fractional PDEs," Physics Letters A, vol. 375, no. 7, pp. 1069-1073, 2011.

[13] S. Guo, L. Mei, Y. Li, and Y. Sun, "The improved fractional sub-equation method and its applications to the space-time fractional differential equations in fluid mechanics," Physics Letters A, vol. 376, no. 4, pp. 407-411, 2012.

[14] B. Lu, "Bäcklund transformation of fractional Riccati equation and its applications to nonlinear fractional partial differential equations," Physics Letters A, vol. 376, no. 28-29, pp. 2045-2048, 2012.

[15] G. Jumarie, "Modified Riemann-Liouville derivative and fractional Taylor series of nondifferentiable functions further results," Computers and Mathematics with Applications, vol. 51, no. 9-10, pp. 1367-1376, 2006.

[16] J. Lee and R. Sakthivel, "New exact travelling wave solutions of bidirectional wave equations," Journal of Physics, vol. 76, no. 6, pp. 819-829, 2011.

[17] L. Song, Q. Wang, and H. Zhang, "Rational approximation solution of the fractional Sharma-Tasso-Olever equation," Journal of Computational and Applied Mathematics, vol. 224, no. 1, pp. 210-218, 2009. 


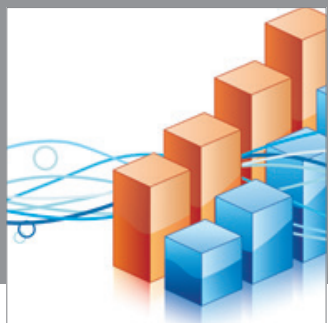

Advances in

Operations Research

mansans

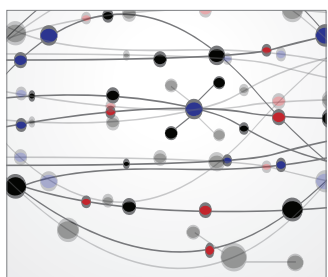

The Scientific World Journal
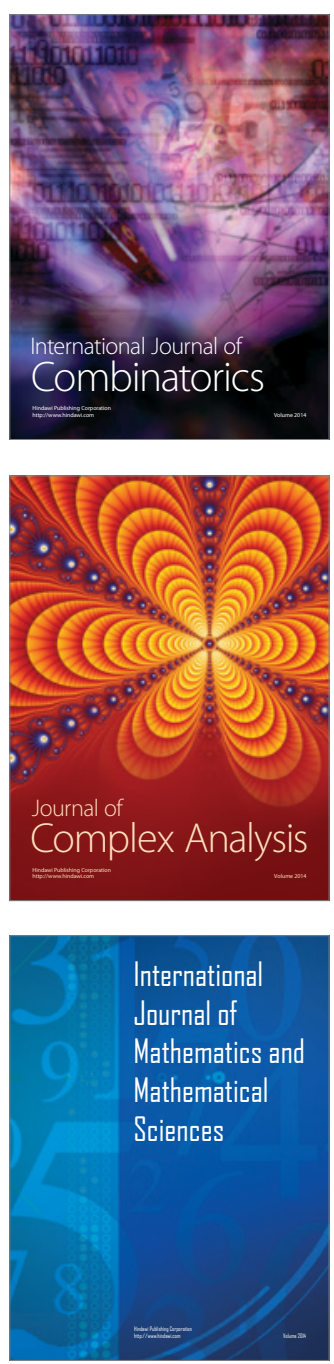
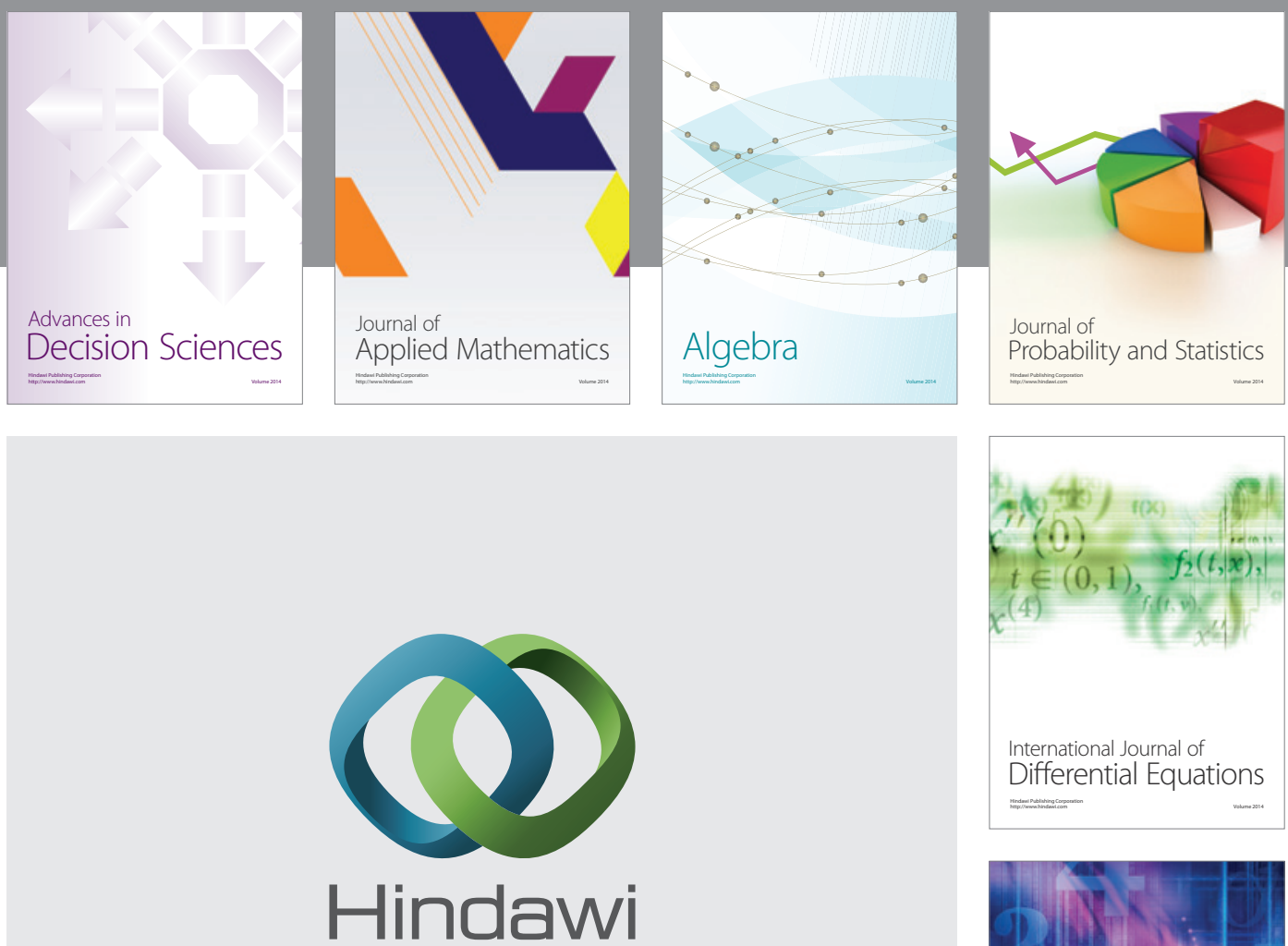

Submit your manuscripts at http://www.hindawi.com
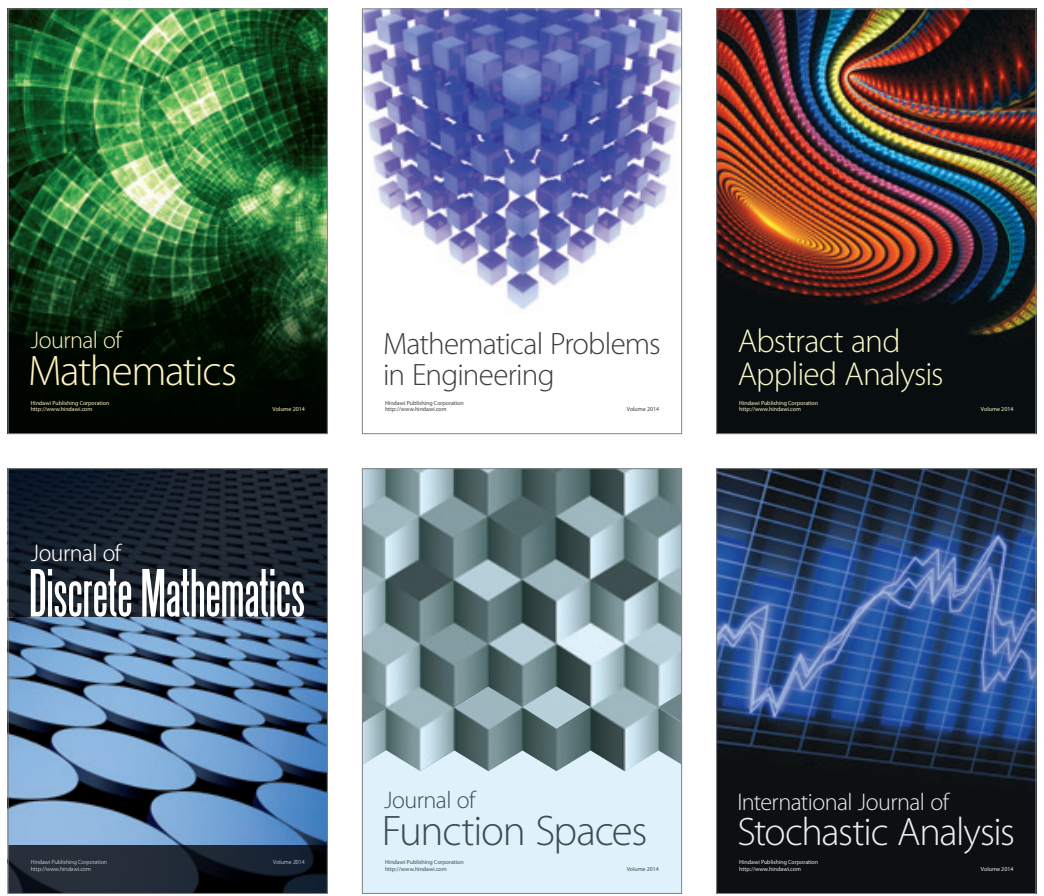

Journal of

Function Spaces

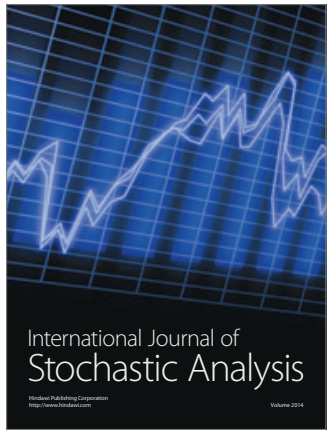

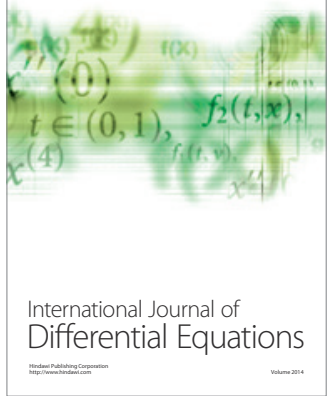
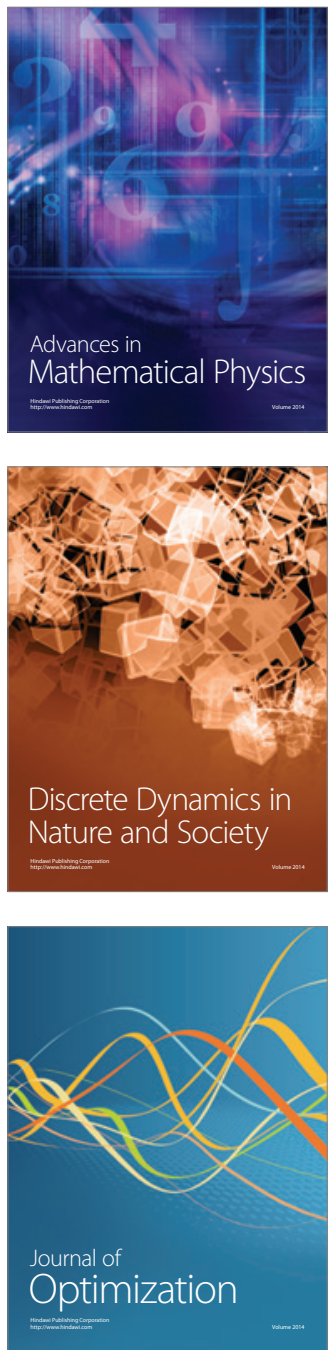